\title{
FLOWS AROUND SUNSPOTS AND PORES
}

\author{
HAIMIN WANG and HAROLD ZIRIN \\ Big Bear Solar Observatory, California Institute of Technology, Pasadena, CA 91125, U.S.A.
}

(Received 12 April, 1991; in revised form 27 January, 1992)

\begin{abstract}
We report on three sequences of high-resolution white-light and magnetogram observations obtained in the summer of 1989. The duration of sub-arcsecond seeing was three to four hours on each day. Study of the white-light and magnetogram data yields the following results:

(1) For all but one of the sunspots we have observed, both dark fibrils and bright grains in the inner part of the penumbra of sunspots move toward the umbra with a speed of about $0.5 \mathrm{~km} \mathrm{~s}^{-1}$. In the outer part of the penumbra, movement is away from the umbra. The one exception is a newly formed spot, which has inflow only in its penumbra.

(2) Granular flows converge toward almost every pore, even before its formation. Pores are observed to form by the concentration of magnetic flux already existing in the photosphere. The pores (or small sunspots), in turn, then move and concentrate to form bigger sunspot.

(3) We followed an emerging flux region (EFR) from 29 to 31 July, 1989 that was composed of a large number of bipoles with magnetic polarities mixed over a large area in the first day of its birth. As time went on, polarities sorted out: the leading polarity elements moved in one direction; the following, the opposite. During the process a large number of cancellations occurred, with some sub-flares and surges observed simultaneously. After about 24 hours, the positive and negative fluxes were essentially separated.

(4) We find two kinds of photospheric dark alignments in the region of new flux emergence: (a) alignments connecting two poles of opposite magnetic polarity form the tops of rising flux tubes; (b) alignments corresponding to the magnetic flux of one polarity, which we call elongated pores.
\end{abstract}

\section{Introduction}

High-resolution observations are essential for solving many problems in solar physics. They have been made from space with the SOUP instrument on board Spacelab II (Title et al., 1986), where the observations are virtually free of atmospheric seeing. Highresolution filtergrams in various photospheric and chromospheric lines have been obtained at Pic du Midi, La Palma and Sac Peak (Muller, 1973, 1983; Muller and Mena, 1987; Brandt et al., 1988; November, 1988; Tarbell et al., 1988). The goal at Big Bear Solar Observatory (BBSO) is to achieve high-resolution images (0.3-0.4 arc sec) near the limit of the telescope modulation transfer function (MTF) for substantial intervals (three to four hours). In a previous paper (Zirin and Wang, 1989), we presented the high-resolution observation of a sunspot and a nearby quiet region obtained on 11 July, 1986. The observation spanned two hours for two targets. In a more recent paper (Zirin and Wang, 1991), we briefly discussed the flow of the 2 August, 1989 sunspot observed in white light, CaK, magnetograms, and Dopplergrams.

In this paper, we present the results of study of the high-resolution white-light data obtained on 17, 29, and 30 July and 2 August, 1989. We discuss the flow pattern of the penumbral material and granules around spots and pores. In addition, we compare the white-light data with high-resolution magnetograph observations from 29 to 31 July, 
1990, in the early stage of an EFR. Magnetograms were obtained almost continuously by BBSO and the Huairou Observatory, Beijing, China during this period.

\section{Data}

High-quality optical images were obtained with the $65-\mathrm{cm}$ vacuum reflector at BBSO, with a Schott VG-9 green filter (bandpass $100 \AA$, center at $5250 \AA$ ). The instrumentation, observation, and data analysis technique were described in detail in the previous paper (Zirin and Wang, 1989). We summarize some of the procedures here.

The continuous video images were recorded on Super-VHS video tapes. The pixel size is typically 0.14 arc sec, giving a field of view of about 90 by 70 arc sec. During the best seeing period, the spatial resolution is limited by the telescope MTF to about 0.3 arc sec. The best seeing periods were from $19: 30$ to $22: 30$ UT 17 July, 17:30 UT 29 July, 16:00 to 18:00 UT 30 July, and 16:20 to 21:20 UT 2 August, 1989. Simultaneous magnetograms, $\mathrm{H} \alpha$, and $\mathrm{CaK}$ filtergrams were also obtained on these days.

We selected and digitized the best video images with the Megavision image processor at a rate of one frame per minute. The selected images were then registered using the maximum correlation technique. They were re-displayed on a video screen and recorded on film.

From 27 July to 1 August, 1989, coordinated magnetograph observations were carried out by BBSO and Huairou Observatory. Big Bear and Huairou have a time difference of about 8 hours, so the two stations together can follow the observing targets almost continuously. The VMG system at BBSO was described in detail by Mosher (1976) and Zirin (1985); the Huairou VMG system was described by Ai (1987). The data combination procedures were discussed by Wang et al. (1989). To achieve a better resolution for magnetograms, the BBSO VMG system was mounted on the $65-\mathrm{cm}$ telescope instead of its usual mounting on the 24-cm telescope. Because of the larger image scale, the magnetograms thus have a pixel size of 0.3 arc sec instead of 0.7 arc sec. Huairou produces 0.7 arc sec pixels at all times.

\section{Velocity Pattern of Granules, Pores, and Sunspots}

We apply correlation tracking (November, 1986; November and Simon, 1988) to track motions of penumbral features and granules. We choose a simple 'box car' shape window with a full width of $1.4 \mathrm{arc} \mathrm{sec}$ for the tracking. The time interval between a pair of images is $5 \mathrm{~min}$. Figures 1 and 2 show the results of the tracking for white-light images of 17 July and 30 July, respectively. Each image contains vectors showing the average velocity for the duration ( 3 and 2 hours, respectively) of each observing period. The background is a corresponding white-light image showing the structure of granules, pores and sunspots. We also divided each observing period into three sub-sections, and plotted the tracking velocity for each section. The velocity patterns are almost the same for all three sections, meaning that the flows do not change much during two to three hours. Generally, the tracking vectors agree with the visual inspection of the movies, 


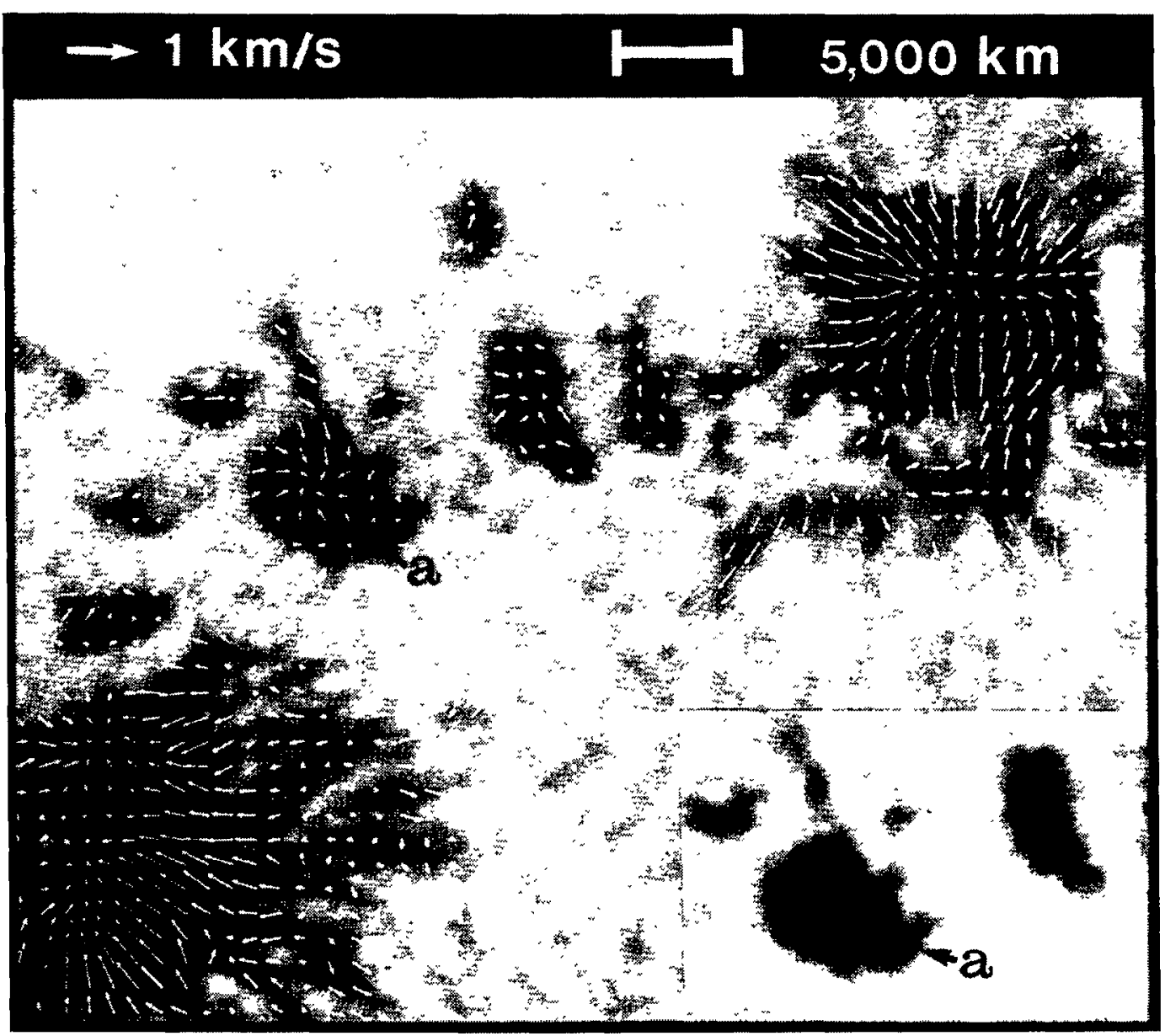

Fig. 1. Flow pattern of active region observed on $17 \mathrm{July}, 1989$ superposed on a white-light image from the middle of the three-hour observing run. Longer-exposure image in lower-right corner shows umbral dots in pore ' $a$ '.

except when features moving in different directions are included in a tracking window, or when there is a transient moving feature in a tracking window.

Based on visual inspections of white-light movies, correlation tracking maps and 'cork movies' (Title et al., 1986) of the active regions, we find the following results on motions in the penumbra and in the area surrounding sunspots.

\subsection{Penumbral flows}

In four of the five largest sunspots we studied, both bright grains and dark fibrils in the inner part of the penumbra move toward the umbra at a speed of about $0.5 \mathrm{~km} \mathrm{~s}^{-1}$. The portion of the penumbra in which inflow occurs ranges from $50 \%$ to $80 \%$ of the total penumbra size. It varies from spot to spot, and from one part of the penumbra to another. The elements in the outer part of the penumbra move outward at about the same speed as the inflow. This produces a divergence in the penumbra. The one exception is the spot shown in Figure 2, where the inflow occurs in $100 \%$ of the penumbra. One difference between the spot in Figure 2 and the two spots in Figure 1 


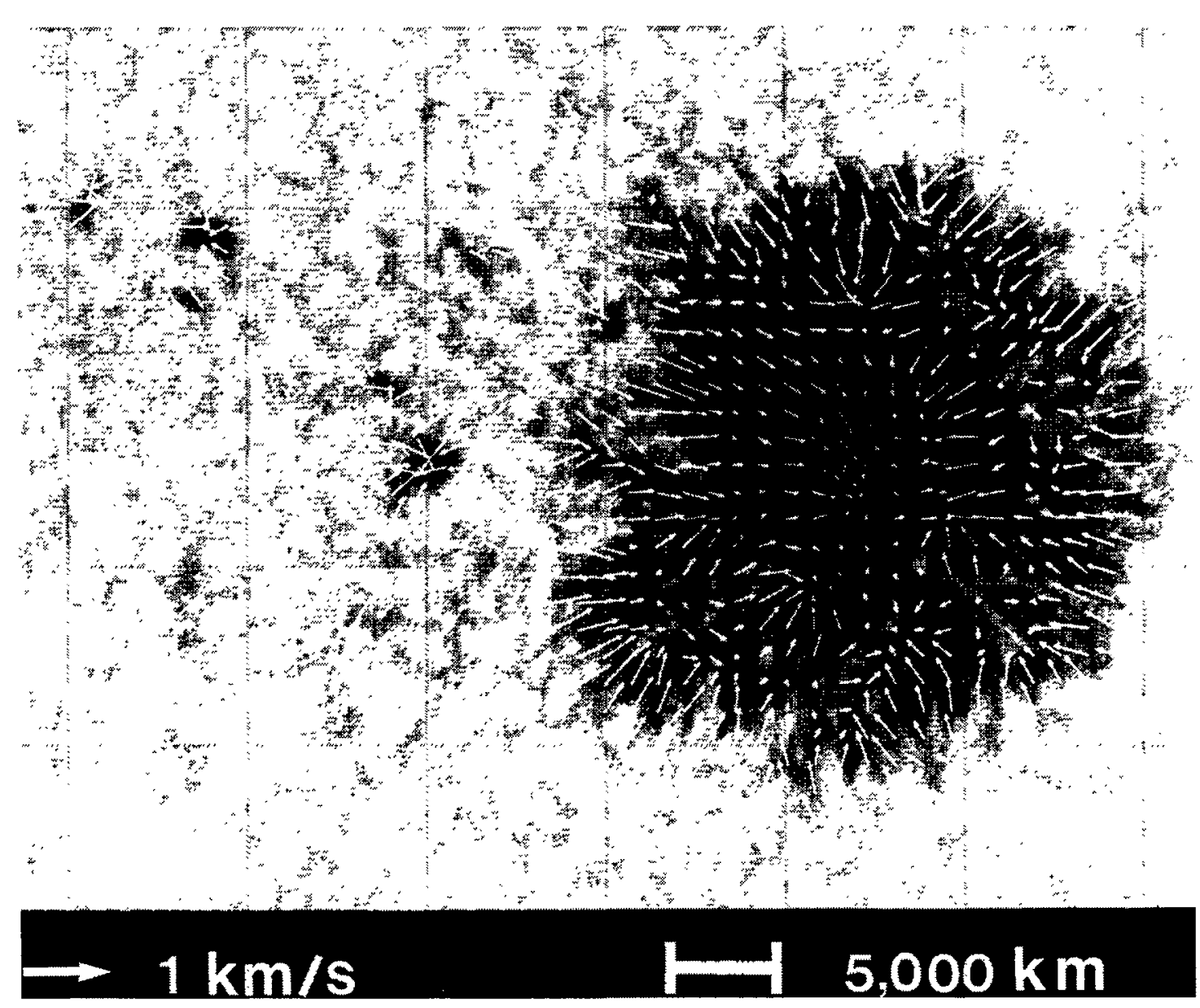

Fig. 2. Flow map and white-light image of the active region observed on $30 \mathrm{July}, 1989$.

is that the former is about 1 day old, the latter, at least 7 days. Is the inflow more dominant in new spots? We need to study more cases to answer this question.

Muller (1973, 1976) and Tönjes and Wöhl (1982) found that in the sunspot penumbra, white grains move inward toward the umbra, the speed of inflow increasing from the outer to the inner part of the penumbra. On the other hand, Schröter (1962) found that bright knots in penumbral filaments move away from the central spot with a speed of 1 to $2 \mathrm{~km} \mathrm{~s}^{-1}$. However, we found that only the observation of the $30 \mathrm{July}$ spot confirms Muller's observation. For other spots, the inflow and outflow depend on the location of the features. Usually, in the inner part of a penumbra everything is moving inward; in the outer part, everything is moving outward. Furthermore, the outflow speed we determine is much less than the value obtained by Schröter. The correlation tracking of the SOUP data (Shine et al., 1986) shows positive divergence which is similar to the pattern obtained here.

In our earlier study (Zirin and Wang, 1991), we found that in white-light and cork movies, the inner part of the penumbra shows inflow; Dopplergrams in that part show outflow. In the outer part of the penumbra, both white-light and Dopplergrams show consistent outflow. 


\subsection{OUTFLOW IN THE SUPER-PENUMBRA}

The super-penumbra is defined as the area of the photosphere immediately outside the penumbra and inside the boundary of the 'moat' (Foukal, 1990) seen in magnetograms. Its width is typically about 5-10 arc sec. The SOUP data showed clearly that all the granules move outward in the super-penumbra. Our observations confirm that.

\subsection{SHRINKING OF A SUNSPOT UMBRA}

The size of the umbra of the 17 July spot (upper-right corner of Figure 1) decreased at a constant rate during the observing period. The total area of the spot decreased about $15 \%$ in three hours. This is one of two shrinking spots we have observed. The other was observed on $21 \mathrm{July}, 1991$, and shrank at a much slower rate.

\subsection{CONVERGING MOTION TOWARD PORES}

While bigger sunspots show an outward motion of granule in their super-penumbra, we find a convergent motion of granules toward pores, which we define as small dark spots

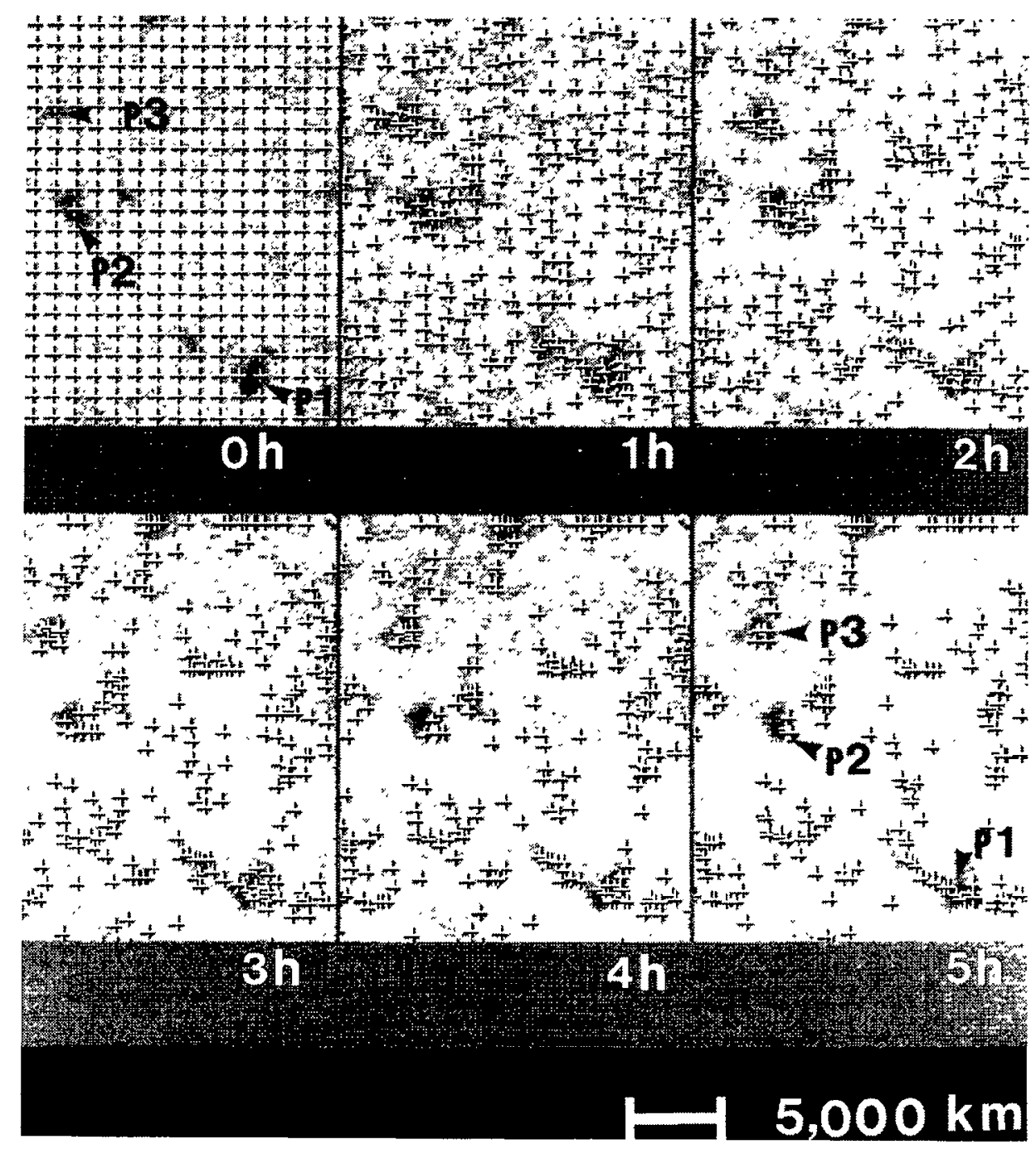

Fig. 3. Time sequence of 'cork' images at 1-hour intervals showing flows converging into pores labeled P1, $\mathbf{P} 2$, and $\mathbf{P} 3$. 
without penumbrae. The convergent inflow has a speed of about $0.5 \mathrm{~km} \mathrm{~s}^{-1}$, and a coherence scale of about 2000 to $3000 \mathrm{~km}$. This flow can be best illustrated as a 'cork movie'. We present a sequence of such images in Figure 3. P1, P2, and P3 are examples of pores showing such inflow. Nine of the 10 pores on 17 and 30 July have such inflow velocity patterns.

\section{New Flux Emergence}

The 30 July 1989 active region (Boulder No. 6233) was the target of a coordinated magnetograph observation run between BBSO and Huairou. The active region was



Fig. 4. Sequence of magnetograms obtained by Huairou and BBSO on 29 and 30 July, showing rapid emergence of new active region. 
followed almost continuously from 00 UT 29 July to 00 UT 2 August, 1989, except for three night gaps of about six hours each. This observing run gave us a good opportunity to follow an EFR from a very early stage of flux emergence. Figure 4 shows a sequence of longitudinal magnetograms covering the first 40 hours of the observing period. Figure 5(a) shows the positive, negative and total magnetic fluxes as a function of time of the EFR; Figure 5(b) shows the separation between two major spots, Pl and F1. The
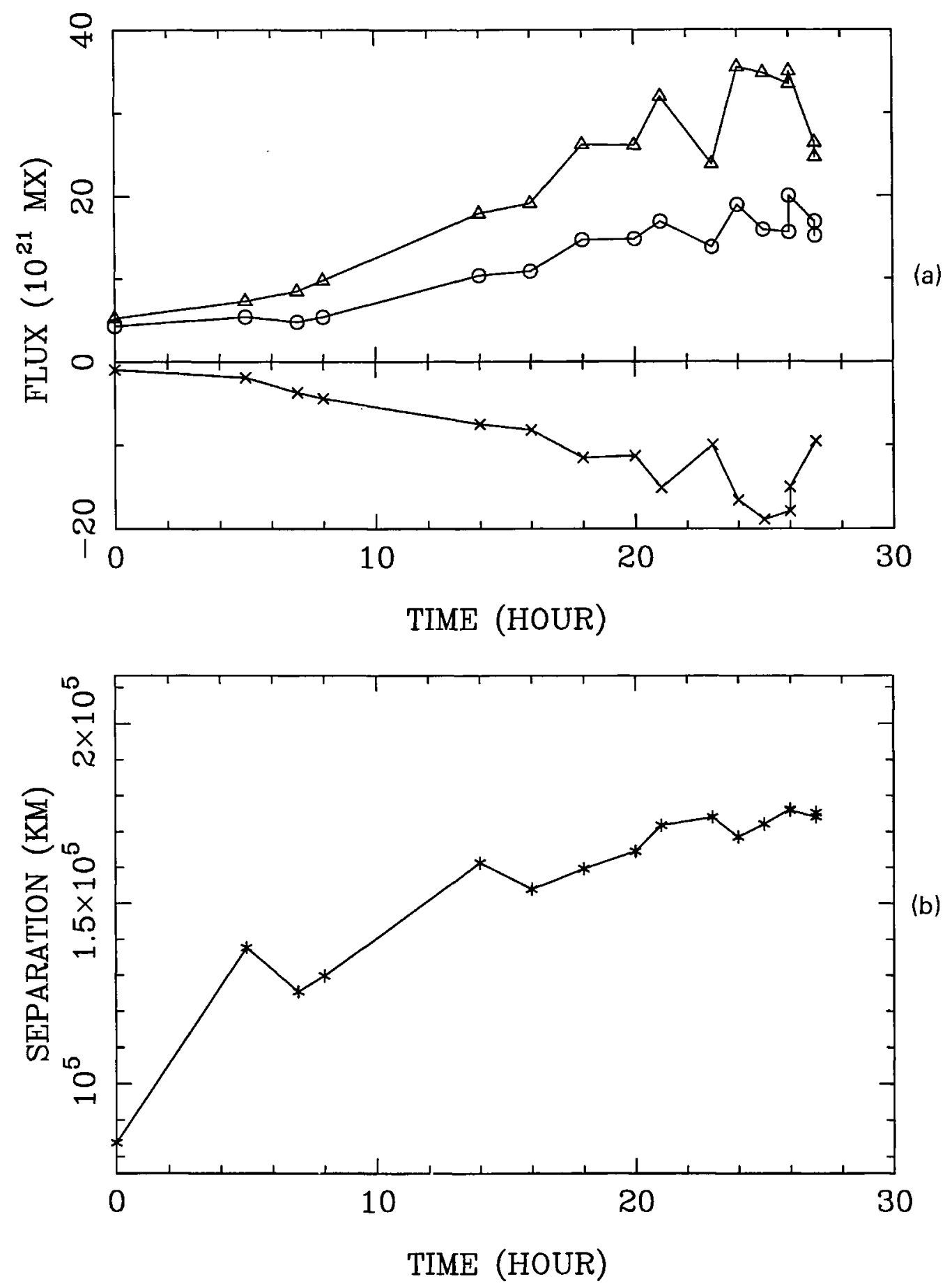

Fig. 5. (a) Negative $(\times \mathrm{s})$, positive (circles), and total (triangles) magnetic flux of the EFR of Figure 4 as function of time. (b) Separation between sunspots P1 and F1 as function of time. 
separation rate starts at about $1.1 \mathrm{~km} \mathrm{~s}^{-1}$ and drops to $0.5 \mathrm{~km} \mathrm{~s}^{-1}$ at the end of the observation.

The initial appearance of the EFR was observed at Huairou from 01 to 07 UT, 29 July, with moderate resolution. An extensive study was made based on BBSO's high-resolution white-light and VMG data from 14 UT 29 July to 00 UT 30 July when the region was still in its early growing stage with a prominent $\mathrm{H} \alpha$ arch filament system. These studies of the EFR have revealed the following:

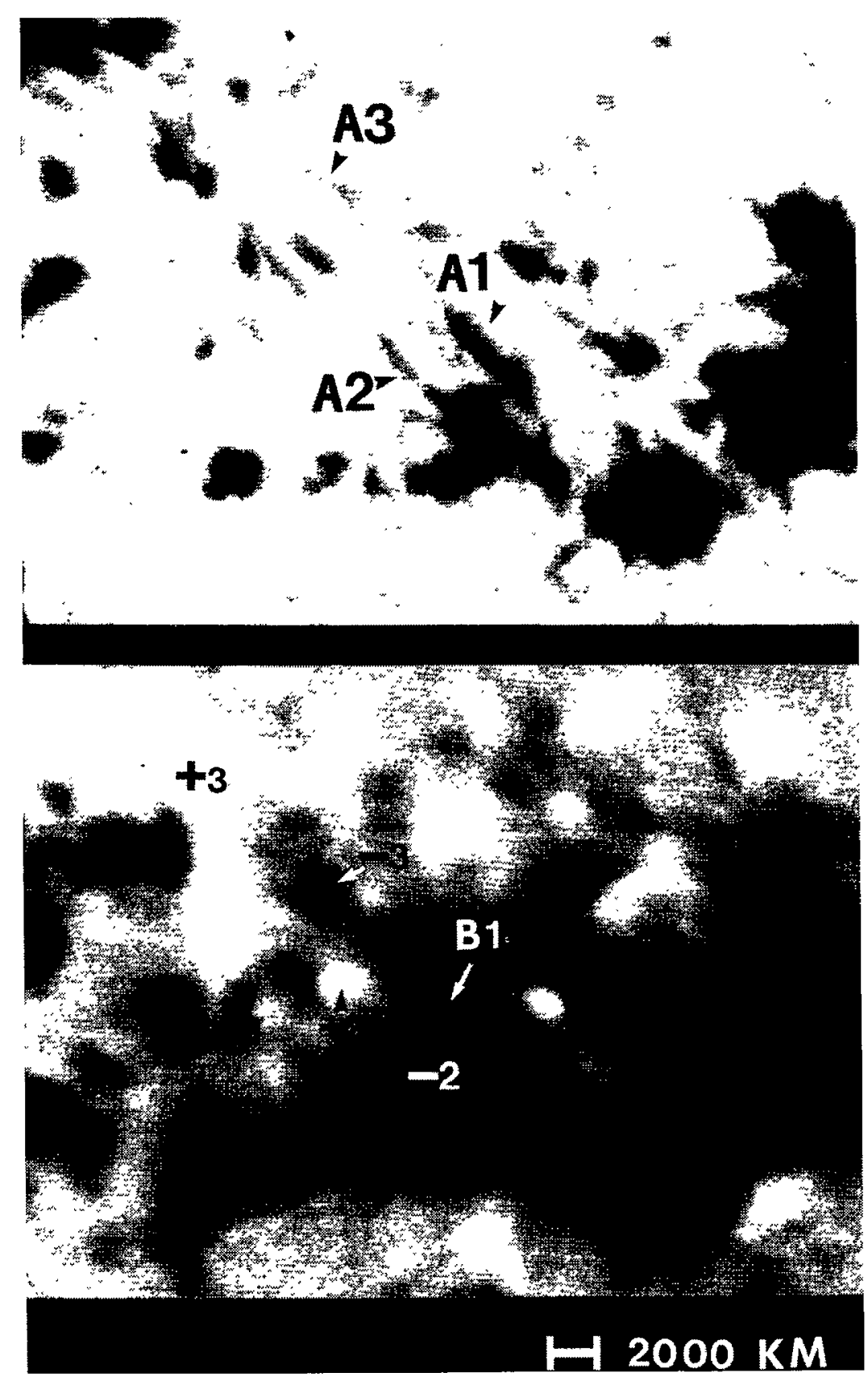

Fig. 6. Comparison between white-light image and magnetogram in the EFR of Figure 4. Dark elongated features ('alignments') either connect two poles with opposite polarity (e.g., A2 and A3) or correspond to strong longitudinal magnetic flux of single polarity (e.g., A1). 
(1) In the area of flux emergence (between P1 and F1, the principal leading and following spots), there exist a large number of bipoles. Since the magnetic fluxes are highly mixed, it is hard to define a neutral line dividing the leading and following polarities. The typical size of the magnetic elements is 1 to $3 \mathrm{Mm}$.

(2) In general, positive and negative fluxes in the new flux emergence area move in opposite directions. This segregating motion implies that flux elements of the same polarity may be rooted in a single source.

(3) Substantial amounts of magnetic flux are cancelled in the process of separating the polarities from the initial mixed stage. A number of sub-flares and surges take place at the same time that we observe these flux cancellations.

(4) Opposite polarities are almost separated after 24 hours. Fluxes in the mixed polarity area have either joined the two major spots, $\mathrm{P} 1$ or $\mathrm{F} 1$, or cancelled out in the process of segregation.

(5) The white-light structure in the area corresponding to the mixed magnetic polarity is similar to the dark alignments observed by Brandt and Steenbeek (1985). In order

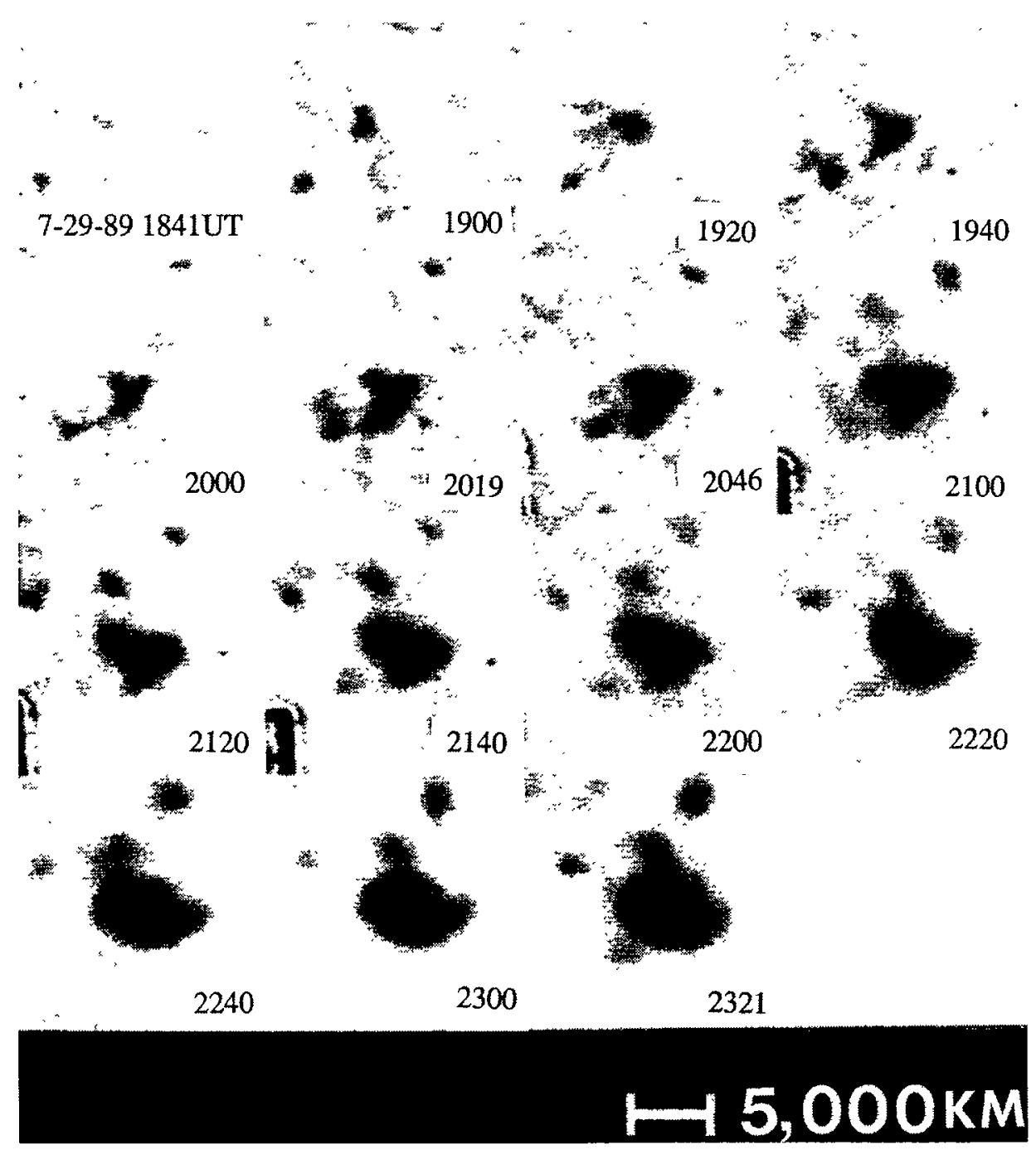

Fig. 7. Five-hour sequence of white-light images showing formation of a pore. 
to study the magnetic properties of the alignments, we compared simultaneous highresolution magnetograms and white-light images. These alignments fall into two categories. The first includes alignments that connect two magnetic elements of opposite polarities. They are probably the tops of rising flux tubes, typically $500 \mathrm{~km}$ wide and $3000 \mathrm{~km}$ long. The magnetic flux of the dipole connected by such an alignment is of order $10^{18} \mathrm{Mx}$. The lifetime of the individual alignment is 10 to $30 \mathrm{~min}$. Thus if the vertical velocity is $1 \mathrm{~km} \mathrm{~s}^{-1}$, the arch thickness is 600 to $2000 \mathrm{~km}$. Most of the alignments belong to this category. A2 and A3 in Figure 6 (obtained at 17:20 UT, 29 July 1989) show examples of such alignments. A2 connects ' +2 ' and ' -2 ' in the magnetogram (below); A3 connects ' +3 ' and ' -3 '. The second category of alignments are those corresponding to strong unipolar longitudinal magnetic fields, which we call elongated pores. A1, corresponding to the magnetic element B 1 in Figure 6, is such an alignment. The second kind of alignments are larger than the first.

If the sunspot is formed by the merging of the smallest observable flux tubes (align-



Fig. 8. Magnetograms corresponding to Figure 7. 
ments), and each alignment has flux of roughly $10^{18} \mathrm{Mx}$, then the spot group we observed must consist of 25000 flux tubes.

In the $\mathrm{H} \alpha$ center line filtergram, we only see relatively large arches (the arch filament system) connecting two major spots (F1-P1). The mixed polarity structure and smallscale alignment structure in the photosphere are underneath the arch filament system. In off-band $\mathrm{H} \alpha$, our spatial resolution is insufficient to see the alignments.

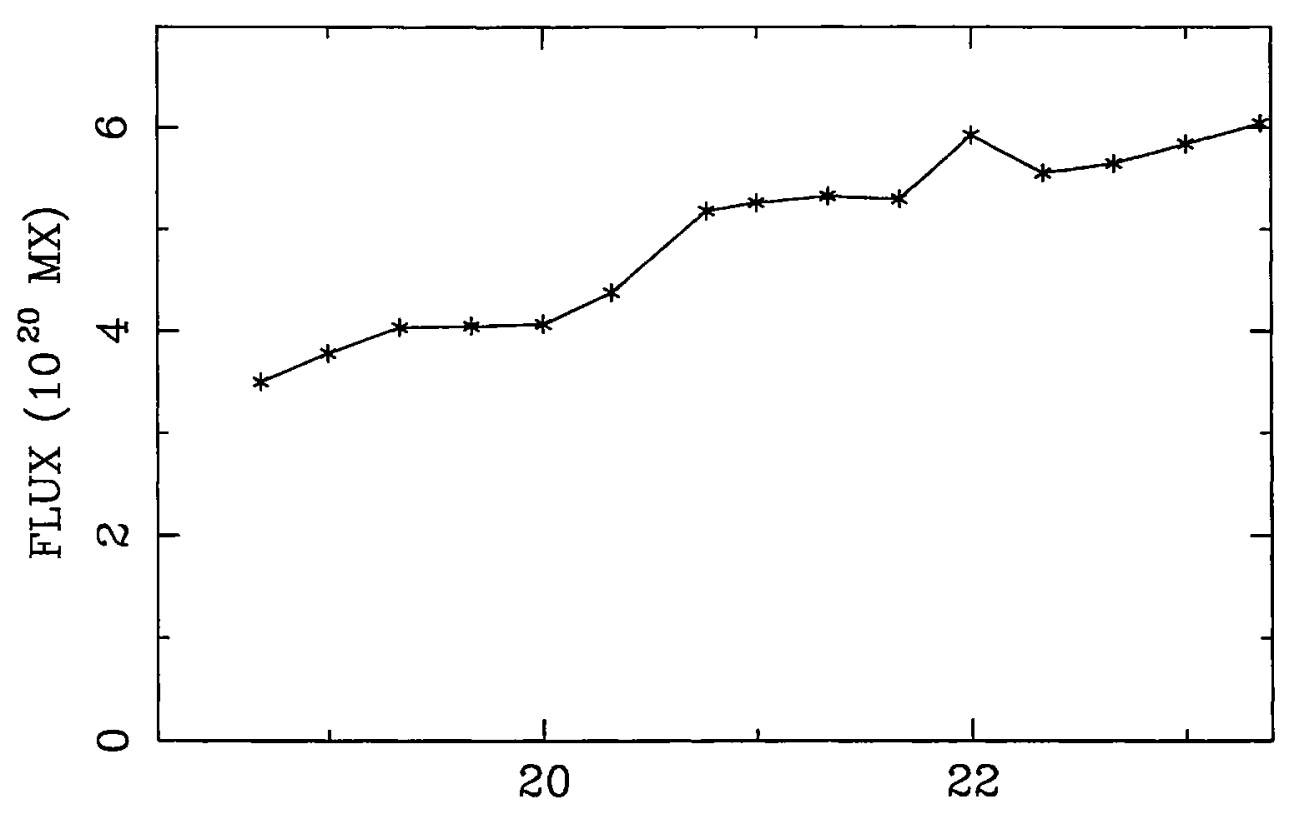

(a)

TIME (UT)

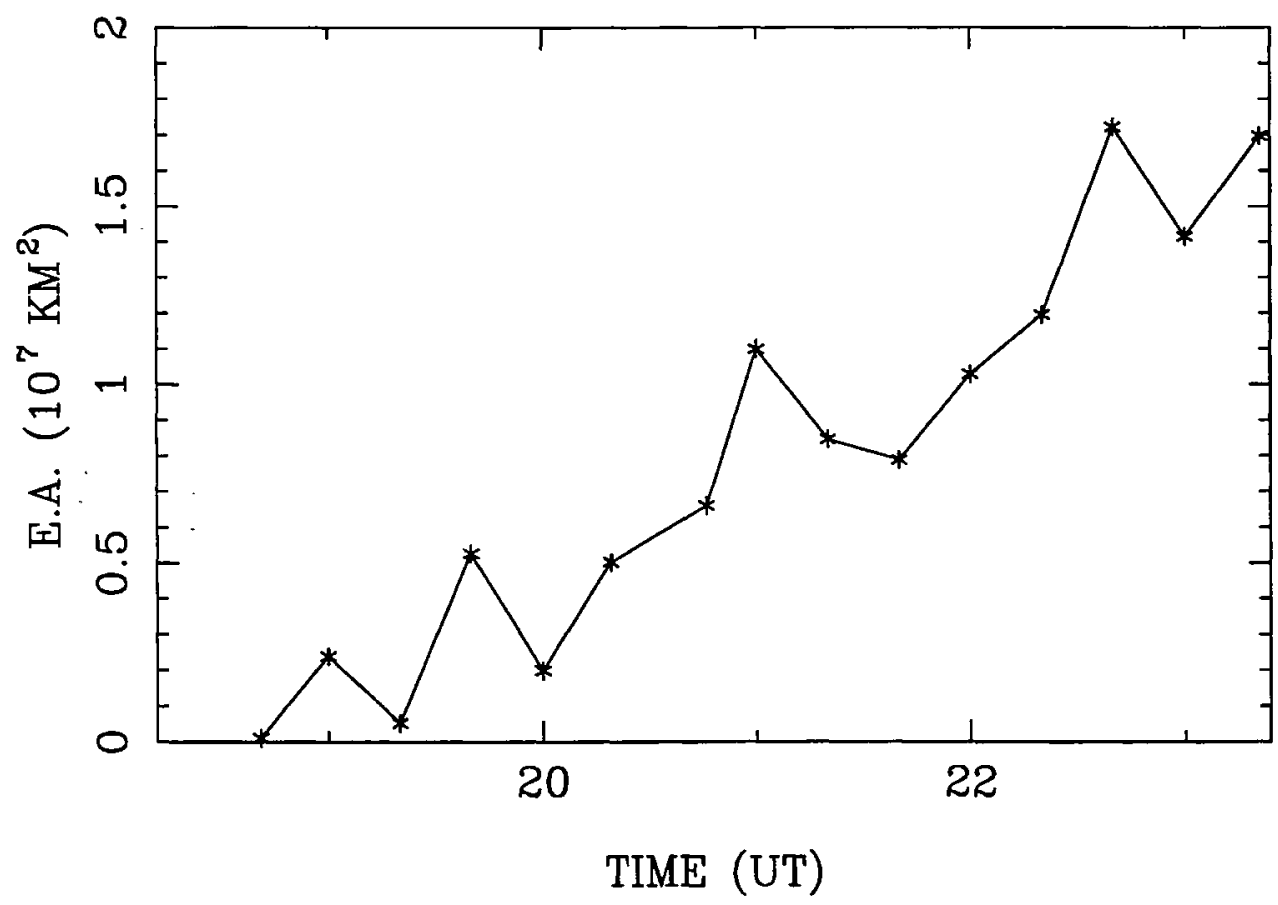

Fig. 9. Time sequences for field-of-view of Figures 7 and 8 showing (a) total negative magnetic flux and (b) equivalent area (cf. text). 
(6) Figure 7 is a sequence of white-light images showing the rapid growth of a small spot (or pore). Figure 8 shows the corresponding growth of the magnetic flux of the spot. Brandt and Steenbeek (1985) studied the evolution of pores in white light and did not see convergence motion toward pores. We, however, do see convergence motion toward pores both in white-light and magnetogram movies. Figures 9(a) and 9(b) show the equivalent area and the magnetic flux of the spot as a function of time. The equivalent area is defined as

$$
\text { E.A. }=\frac{\left(I_{0}-I_{p}\right)}{I_{0}} S,
$$

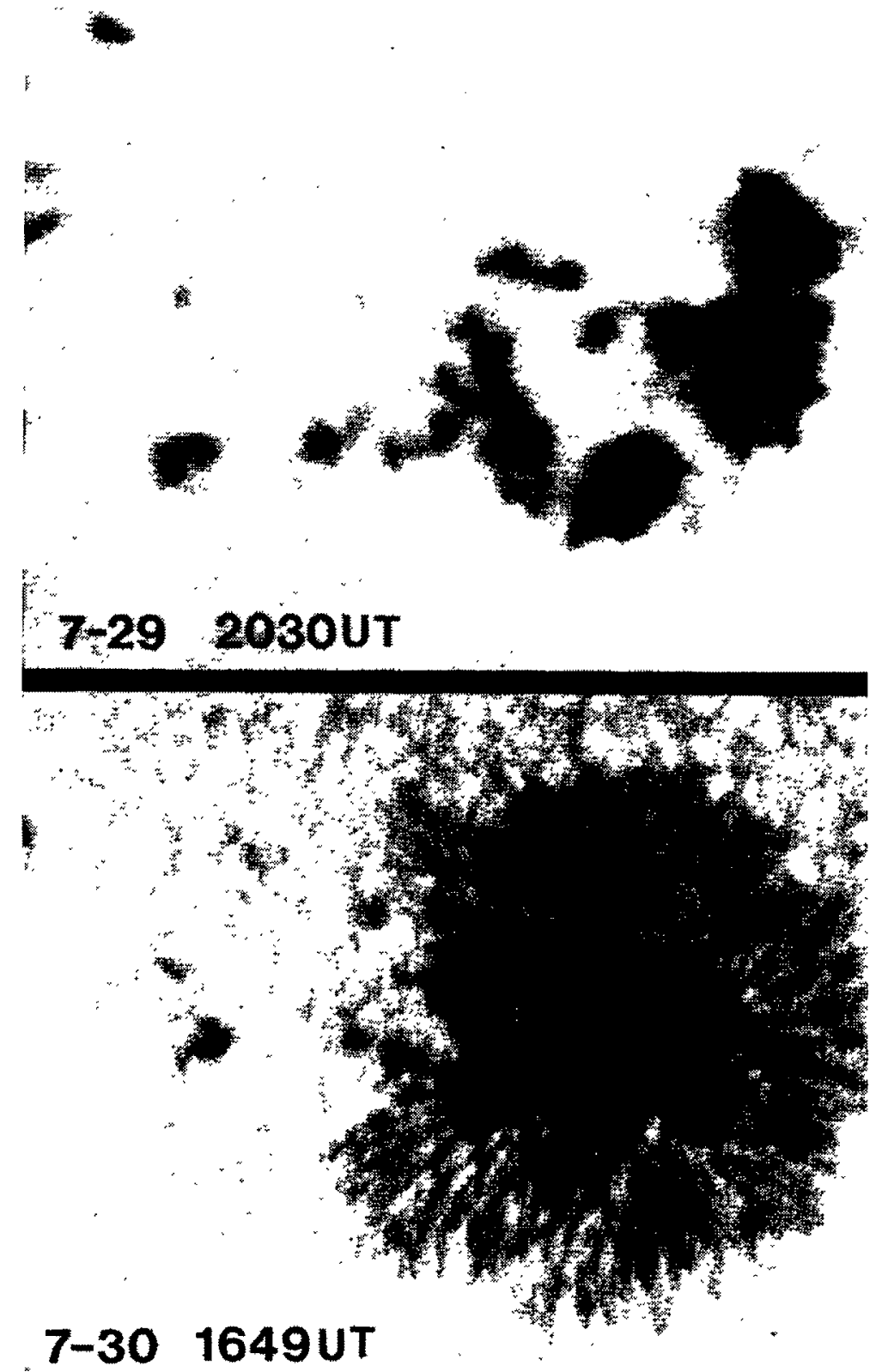

Fig. 10. White-light images taken about 20 hours apart, showing formation of a big sunspot from many smaller spots. Light bridges may divide merged smaller spots. 
where $I_{0} \mathrm{i}$ the average quiet-Sun intensity and $I_{p}$ is the average intensity of the field-ofview which has an area $S$ (20 arc sec by 20 arc sec). From Figure 9 we see that more than $50 \%$ of the magnetic flux of the formed spot already existed in the field-of-view at the beginning of the observing run when the equivalent area of the spot was still zero.

(7) The major $p$ and $f$ spots are formed by merging of smaller spots. Unfortunately, there is a big night gap between the BBSO high-resolution observations of 29 and 30 July. Figure 20 shows two images about 20 hours apart. The merging of the sunspots may be related to the complicated light bridge system seen on 30 July, but we do not have the evidence for it due to the night gap. However, we see that two light bridges are forming while the spots are merging during the continuous observation of 29 July.

\section{Summary}

Analysis of high-resolution white-light and magnetograph data obtained in the summer of 1989 on three active regions reveals the following:

(1) Both inflow and outflow are observed in the penumbrae of most spots. Somewhere in the penumbra, there usually exists a ring-like boundary. Inside this boundary, both bright grains and dark fibrils move inward toward the umbra; outside the boundary all the material moves outward. Both inflow and outflow in the penumbra have a speed of about $0.5 \mathrm{~km} \mathrm{~s}^{-\mathrm{I}}$. For the $30 \mathrm{July}, 1989$ spot, this ring-like boundary is located at the photosphere-penumbra border, so the whole penumbra has only inflow. Because this result is in contradiction to the earlier work of Muller (1973) and Schröter (1962), we are in the process of studying more data obtained in 1990 and 1991 to see if other examples show the same characteristics.

(2) Complicated processes occur in an emerging flux region. There exist numerous small flux tubes with magnetic flux of order $10^{18} \mathrm{Mx}$ in the area of new flux emergence. In the magnetograph and white-light movies, flux elements move and merge to form small sunspots. Small sunspots then merge into larger ones. In the area where the small flux tubes emerge, magnetic polarities are initially highly mixed. Elements with different polarities move in opposite directions. During the process of segregation, flux cancellation occurs continuously, thus removing part of the flux from the emerging active region. Such cancellations may trigger sub-flares and surges observed simultaneously. The merging of flux tubes to form sunspots agrees with magnetoconvection models by several authors (e.g., Galloway and Weiss, 1981; Parker, 1982; Schmidt, Simon, and Weiss, 1985). Those models demonstrate how magnetic flux can be concentrated into isolated ropes in the convection zone. However, the existence of mixed magnetic polarity in the area of flux emergence suggests that the sub-surface fields are highly tangled.

\section{Acknowledgements}

We are grateful to the observing staff at BBSO and at Huairou Solar Observing Station for their support in the observations. We thank the referee for helpful comments and criticism. We are also grateful to Frances Tang, Sara Martin, and Leila Belkora for 
reading the manuscript and helpful comments. This work is supported by NSF under grant ATM-8816007 and by NASA under grants NAGW-1972 and NASG-27269.

\section{References}

Ai, G.: 1987, Publ. Beijing Astron. Obs. 9, 27.

Brandt, J. J. and Steenbeek, J. C. M.: 1985, Solar Phys. 96, 229.

Brandt, P. N., Ferguson, S., Scharmer, G. B., Shine, R. A., Tarbell, T. D., Title, A. M. and Topka, K.: 1988, in O. Von der Luhe (ed.), High Resolution Solar Observations, Proceedings of Sac Peak Summer Workshop, National Solar Observatory, Sunspot, NM, p. 473.

Foukal, P.: 1990, Solar Astrophysics, Wiley Interscience, New York.

Galloway, D. J. and Weiss, N. O.: 1981, Astrophys. J. 243, 945.

Mosher, J. M.: 1976, BBSO Preprint.

Muller, R.: 1973, Solar Phys. 29, 55.

Muller, R.: 1976, Solar Phys. 48, 101.

Muller, R.: 1983, Solar Phys. 85, 113.

Muller, R. and Mena, B.: 1987, Solar Phys. 112, 295.

November, L.: 1986, Appl. Optics 25, 392.

November, L.: 1988, in O. Von der Luhe (ed.), High Resolution Solar Observations, Proceedings of Sac Peak Summer Workshop, National Solar Observatory, Sunspot, NM, p. 457.

November, L. J. and Simon, G.: 1988, Astrophys. J. 333, 427.

Parker, G. E.: 1982, Astrophys. J. 256, 292.

Schmidt, H. U., Simon, G. W., and Weiss, N. O.: 1985, Astron. Astrophys. 148, 191.

Schröter, E. H.: 1962, Z. Astrophys. 56, 183.

Shine, R. A., Title, A., Tarbell, T. and the SOUP Team: 1986, Proceedings of the Second Workshop on Problems in High Resolution Solar Observation, Boulder, CO.

Tarbell, T., Ferguson, S., Frank, Z., Title, A., and Topka, K.: 1988, in O. Von der Luhe (ed.), High Resolution Solar Observations, Proceedings of Sac Peak Summer Workshop, National Solar Observatory, Sunspot, NM, p. 506.

Title, A., Tarbell, T., Simon, G. and the SOUP Team: 1986, Adv. Space Res. 6, 253.

Tönjes, K. and Wöhl, H.: 1982, Solar Phys. 75, 63.

Wang, H., Zirin, H., Patterson, A., Ai, G., and Zhang, H.: 1989, Astrophys. J. 343, 489.

Zirin, H.: 1985, Australian J. Phys. 38, 961.

Zirin, H. and Wang, H.: 1989, Solar Phys. 119, 245.

Zirin, H. and Wang, H.: 1991, Space Rev. 11, 225. 